\title{
Exhaled nitric oxide in stable adult cystic fibrosis patients, during exacerbation and following CFTR-modifying treatment
}

\author{
To the Editor:
}

In adult patients with cystic fibrosis (CF) fraction of exhaled nitric oxide ( $F$ eNO) has been reported to be normal or abnormally low [1]. FeNO has also been reported to correlate with spirometry [2-4], and to increase following treatment with ivacaftor [5, 6], suggesting that FeNO could be used as a noninvasive, fast and easily available marker of CFTR-function. The aim of this study was to examine whether in adult CF patients, FeNO was associated with patient characteristics and lung function, and whether FeNO was affected by acute exacerbation. We also aimed to examine FeNO evolution over a longer period after starting cystic fibrosis transmembrane conductance regulator (CFTR)-modifying treatment (ivacaftor and ivacaftor/lumacaftor).

We conducted a prospective observational study (December 2015-December 2017) at the CF Reference Centre of the University Hospital of Brussels, measuring FeNO and standard lung function in adult CF patients in a stable condition (at $~ 3$-monthly intervals) as well as during acute exacerbation, as defined by Bilton et al. [7]. We recorded sex, age, smoking status, genotype, pancreatic function, presence of atopy and/or coexistent asthma, and presence of colonisation. For the subgroup of CF patients without any factor known to confound FeNO (atopy, coexistent asthma, active smoking, chronic treatment with corticosteroids), a control group of age- and sex-matched (1:1) healthy subjects was recruited from healthy never-smokers. In patients receiving CFTR-modifying treatment (ivacaftor or ivacaftor/lumacaftor) FeNO was measured prior to the start of treatment, and at regular intervals during a follow-up period of 12 months. Log-transformed FeNO values were used for comparisons between groups or subgroups (unpaired t-tests), or for comparison between a stable condition and acute exacerbation (paired t-test). Normality of the log-transformed FeNO values as well as of the residuals (in comparative tests) was verified with the Chi-squared test. Rank correlations (Spearman) between FeNO and lung function or body mass index (BMI) were also performed, using Medcalc (version 18; MedCalc Software, Ostend, Belgium).

In total, 62 adult $\mathrm{CF}$ patients were eligible, which included 17 patients without any factor known to confound FeNO and seven patients on CFTR-modifying treatment (ivacaftor or ivacaftor/lumacaftor). A total of 264 FeNO measurements were collected, in a stable condition as well as at onset of acute exacerbation (prior to antibiotic treatment). Median (95\% CI) age was 30 (26-33) years and 68\% were men, median stable forced expiratory volume in $1 \mathrm{~s}$ (FEV1) was $2.50 \mathrm{~L}$ (71\% predicted). 39\% were homozygous for the F508del mutation. 48\% were colonised with Pseudomonas aeruginosa and $82 \%$ were colonised with meticillin-sensitive Staphylococcus aureus (MSSA); only 5\% were colonised with meticillin-resistant $S$. aureus (MRSA). From the total patient group of 62 patients, 55 showed pancreatic insufficiency (89\%). Compared with healthy controls (geometric mean (95\% CI) FeNO 16.7 (11.8-23.7) ppb), the patient subgroup without FeNO confounders showed a significantly lower FeNO (10.7 (9.2-13.0) ppb; $\mathrm{p}=0.028)$. In the entire CF patient group, geometric mean FeNO was 8.9 (7.8-10.3) ppb. In patients colonised with P. aeruginosa, FeNO was lower (geometric mean 7.0 (5.6-8.8) ppb) compared to those who were not $(11.1(9.6-12.9) \mathrm{ppb} ; \mathrm{p}<0.001)$. Receiver operating curve-analysis showed an area under the curve of $76 \%$, with a specificity of $81 \%$ and a sensitivity of $70 \%$ for presence of $P$. aeruginosa (FeNO cut-off: $8.8 \mathrm{ppb}$ ). In patients colonised with MSSA, FeNO was higher (geometric mean (95\% CI) 9.7 $(8.4-11.2) \mathrm{ppb})$ compared to those who were not $(6.2(4.2-9.2) \mathrm{ppb} ; \mathrm{p}=0.014)$. We found no association of

$@$ ERSpublications

FeNO is low in stable adult patients with CF, and lower in patients colonised with $P$. aeruginosa. FeNO cannot be used as a noninvasive marker of CFTR function, since prolonged follow-up showed only a transient rise in FeNO after starting ivacaftor. http://ow.ly/PtfC30ojYFC

Cite this article as: Vincken S, Verbanck S, De Wachter E, et al. Exhaled nitric oxide in stable adult cystic fibrosis patients, during exacerbation and following CFTR-modifying treatment. Eur Respir J 2019; 53: 1802259 [https://doi.org/10.1183/13993003.02259-2018]. 
FeNO with other clinical characteristics such as genotype F508del/F508del, pancreatic insufficiency or BMI. None of the lung function parameters (FEV1, forced vital capacity, residual volume/total lung capacity, transfer coefficient of the lung for carbon monoxide) correlated with $F e N O(p>0.1$ for all). In the 52 patients with an acute exacerbation (geometric mean (95\% CI) 10.1 (8.1-12.5) ppb), FeNO was similar to that in a stable condition $(8.9(7.6-10.4) \mathrm{ppb} ; \mathrm{p}=0.16)$. In the three patients receiving ivacaftor, there was an initial rise in FeNO followed by a return towards pretreatment values 8-12 months after the start of CFTR-modifying treatment (figure 1a). In particular, FeNO increased from 6.7 to $16.3 \mathrm{ppb}$, from 10.4 to $18.6 \mathrm{ppb}$ and from 15.5 to $26.9 \mathrm{ppb}$. In three out of the four patients receiving ivacaftor/lumacaftor, an initial increase could be observed in FeNO: from 8.9 to $15.4 \mathrm{ppb}$, from 12.2 to $14.0 \mathrm{ppb}$ and from 16.6 to $19.9 \mathrm{ppb}$; yet, in one patient FeNO decreased from 15.5 to $12.3 \mathrm{ppb}$ (figure 1b).

FeNO in CF patients has been described as either lower than, or not different from, that in healthy controls [1]. Differences in measuring methodology, small patient groups and confounders such as corticosteroid therapy may have contributed to these inconsistent results. In this prospective observational study on a substantial number of adult CF patients, in whom FeNO was measured multiple times over a 2-year period, we demonstrate that FeNO is consistently low in stable patients. Since CF is characterised by chronic neutrophilic airway inflammation, one could assume that FeNO, generally used as a marker of eosinophilic airway inflammation, is indeed not increased in CF patients. On the contrary, FeNO is decreased in stable CF patients. There are several possible explanations for this finding. Modelling of perturbed nitric oxide diffusion through a thickened mucus lining of the airways predicts a distinct FeNO decrease, even when nitric oxide production is unaltered [8]. Malnutrition/malabsorption may result in a lack of L-arginine, the substrate for nitric oxide production $[1,9,10]$. In patients with $\mathrm{CF}$, increased levels of asymmetric dimethyl arginine [10], the endogenous inhibitor of cellular arginine uptake and nitric oxide synthase (NOS) activity, and the inability to upregulate inducible NOS (iNOS) despite the presence of chronic inflammation $[1,9,10]$ may also be responsible. Finally, the consumption of nitric oxide by P. aeruginosa and other denitrifying organisms $[1,9,10]$, as well as variants in the genes encoding constitutive NOS, which have been associated with low FeNO and colonisation with $P$. aeruginosa $[11,12]$, could explain the significantly lower FeNO we observed in patients colonised with P. aeruginosa. Hence, a low FeNO may signal $P$. aeruginosa colonisation, and as such, be prognostic of disease progression. This hypothesis would need to be confirmed by a prospective trial following up patients with $P$. aeruginosa colonisation. We also detected an association between the presence of MSSA colonisation and higher FeNO, but this observation may have been biased by the uneven distribution (51 with versus 11 patients without MSSA colonisation).

A subject of controversy is whether reduced iNOS expression is primarily related to reduced or absent CFTR function, or due to chronic inflammation. The hypothesis of a link between decreased CFTR function and lower iNOS expression is interesting, since it implies that FeNO could be used as a noninvasive marker of CFTR-function, as suggested by two studies [5, 6]. In 15 CF patients (eight adults and seven children) [5] and in five children with CF [6] these authors showed FeNO increases 4 weeks after the start of treatment with ivacaftor, a CFTR-modifying drug, and this effect was more pronounced in the

a)

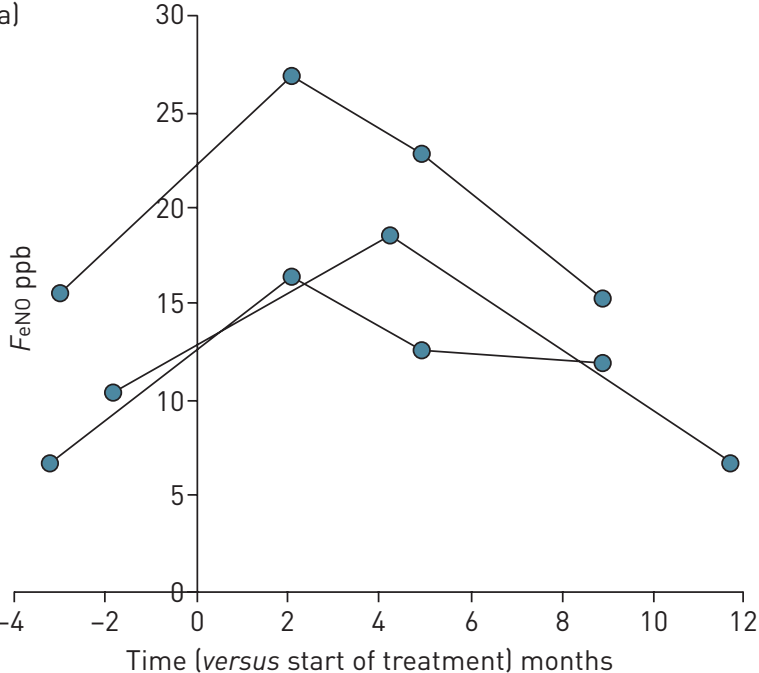

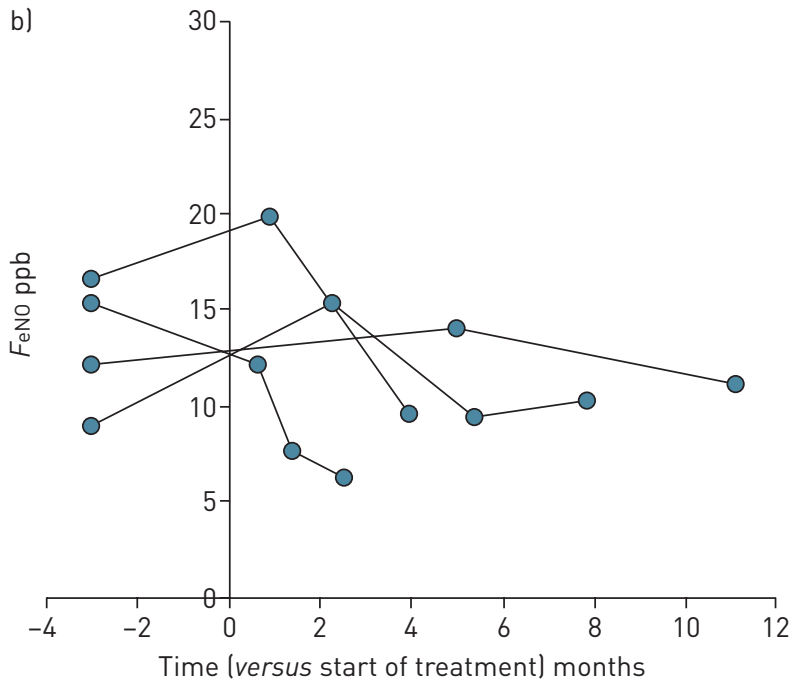

FIGURE 1 a) Evolution of fraction of exhaled nitric oxide $\left(F_{\mathrm{eNO}}\right)$ in the three patients started on treatment with ivacaftor during the study period. b) Evolution of $F$ eNO in the four patients started on treatment with ivacaftor/lumacaftor during the study period. Zero on the time axis indicates the start of treatment with a) ivacaftor or b) ivacaftor/lumacaftor. 
paediatric cohort. We also observed an initial rise in FeNO in each of the three adult patients in the first few months after starting treatment with ivacaftor. However, this was a transient effect, and FeNO values were back to baseline values by 8-12 months after the start of treatment. Over this period, none of the three patients experienced an acute exacerbation, lung function decline (rather the opposite) or other health-related problems. Changes in FeNO after starting ivacaftor/lumacaftor in patients homozygous for F508del were less consistent compared with the observed FeNO changes in the patients receiving ivacaftor treatment. A possible explanation is that ivacaftor/lumacaftor only partially rescues CFTR function in these patients, as also supported by the more modest and variable clinical changes (in sweat chloride and FEV1) seen in association with this treatment [13]. To our knowledge, our study is the first to follow up FeNO for a period exceeding 1 month after the start of ivacaftor treatment, and the first study to monitor FeNO during treatment with ivacaftor/lumacaftor over a comparable follow-up period of up to 11 months after the start of treatment.

In conclusion, in this prospective observational study spanning 2 years, we reported consistently low FeNO values in stable adult patients with CF. FeNO was even lower in patients with P. aeruginosa colonisation, suggesting that FeNO monitoring could be used as a marker of $P$. aeruginosa colonisation and as such be prognostic for disease progression. Taking into account the limited patient numbers studied, and thus using caution interpreting these results, our observations do not support the hypothesis that FeNO can be used as a noninvasive marker of CFTR function, since prolonged follow-up of FeNO after the start of CFTR-modifying treatment showed a return of FeNO towards pretreatment values after a transient rise in the patients treated with ivacaftor, and no consistent changes in the patients treated with ivacaftor/ lumacaftor.

Stefanie Vincken $\oplus^{1}$, Sylvia Verbanck ${ }^{1}$, Elke De Wachter ${ }^{2}$ and Eef Vanderhelst ${ }^{1}$

${ }^{1}$ Division of Respiratory Diseases, Dept of Medicine, Universitair Ziekenhuis Brussel, Vrije Universiteit Brussel, Brussels, Belgium. ${ }^{2}$ Dept of Paediatrics, Universitair Ziekenhuis Brussel, Vrije Universiteit Brussel, Brussels, Belgium.

Correspondence: Stefanie Vincken, Division of Respiratory Diseases, Dept of Medicine, Universitair Ziekenhuis Brussel, Vrije Universiteit Brussel, Laarbeeklaan 101, 1090 Jette, Belgium. E-mail: stefanie.vincken@uzbrussel.be

Received: Nov 282018 | Accepted after revision: Feb 202019

Conflict of interest: None declared.

\section{References}

1 de Winter-de Groot K, van der Ent CK. Nitric oxide in cystic fibrosis. J Cyst Fibros 2005; 4: Suppl. 2, $25-29$.

2 Ho LP, Innes JA, Greening AP. Exhaled nitric oxide is not elevated in the inflammatory airways diseases of cystic fibrosis and bronchiectasis. Eur Respir J 1998; 12: 1290-1294.

3 Krantz C, Janson C, Hollsing A, et al. Exhaled and nasal nitric oxide in relation to lung function, blood cell counts and disease characteristics in cystic fibrosis. J Breath Res 2017; 11: 026001.

4 Keen C, Gustafsson P, Lindblad A, et al. Low levels of exhaled nitric oxide are associated with impaired lung function in cystic fibrosis. Pediatr Pulmonology 2010; 45: 241-248.

5 Grasemann H, Gonska T, Avolio J, et al. Effect of ivacaftor therapy in exhaled nitric oxide in patients with cystic fibrosis. J Cyst Fibros 2015; 14: 727-732.

6 Kotha K, Szczesniak RD, Naren AP, et al. Concentration of fractional excretion of nitric oxide (FeNO): a potentia airway biomarker of restored CFTR function. J Cyst Fibros 2015; 14: 733-740.

7 Bilton D, Canny G, Conway S, et al. Pulmonary exacerbation: towards a definition for use in clinical trials. Report from the EuroCareCF Working Group on outcome parameters in clinical trials. J Cyst Fibros 2011; 10: Suppl. 2, S79-S81.

8 Karamaoun C, Haut B, Van Muylem A. A new role for the exhaled nitric oxide as a functional marker of peripheral airway caliber changes: a theoretical study. J Appl Physiol 2018; 124: 1025-1033.

9 Grasemann H, Ratjen F. Cystic fibrosis lung disease: The role of nitric oxide. Pediatr Pulmonology 1999; 28: $442-448$.

10 Marozkina NV, Gaston B. Nitrogen chemistry and lung physiology. Annu Rev Physiol 2015; 77: $431-452$.

11 Grasemann H, Knauer N, Büscher R, et al. Airway nitric oxide levels in cystic fibrosis patients are related to a polymorphism in the neuronal nitric oxide synthase gene. Am J Respir Crit Care Med 2000; 162: 2172-2176.

12 Grasemann H, Storm van's Gravesande K, Buscher R, et al. Endothelial nitric oxide synthase variants in cystic fibrosis lung disease. Am J Respir Crit Care Med 2003; 167: 390-394.

13 Wainwright CE, Elborn JS, Ramsey BW, et al. Lumacaftor-ivacaftor in patients with cystic fibrosis homozygous for Phe508del CFTR. N Engl J Med 2015; 373: 220-231. 